\title{
Perimeter Crystallization of Amorphous Silicon around a Germanium Seed
}

\author{
M. M. A. Hakim, ${ }^{\text {a, }}$ I. Matko, ${ }^{\text {b }}$ B. Chenevier, ${ }^{\text {b }}$ and P. Ashburn ${ }^{\text {a }}$ \\ ${ }^{a}$ School of Electronics and Computer Science, University of Southampton, Southampton, United Kingdom \\ ${ }^{b}$ Laboratoire des Materiaux et du Genie Physique, Ecole Nationale Superieure de Physique de \\ Grenoble, Grenoble, France
}

\begin{abstract}
An $\alpha$-Si crystallization phenomenon that originates from the perimeter of a germanium seed during anneal at $500^{\circ} \mathrm{C}$ is reported The perimeter crystallization is reasonably uniform and reaches $500 \mathrm{~nm}$ after a $60 \mathrm{~h}$ anneal at $500^{\circ} \mathrm{C}$. Transmission electron microscopy shows that the crystallized material is polycrystalline and made up of grains with various orientations. In contrast, $60 \mathrm{~h}$ anneal at $550{ }^{\circ} \mathrm{C}$ gives increased $\alpha$-Si crystallization beneath the entire germanium seed, as reported previously. This perimeter crystallization phenomenon is advantageous because it allows $\alpha$-Si crystallization to be achieved at a lower thermal budget.
\end{abstract}

(c) 2006 The Electrochemical Society. [DOI: 10.1149/1.2199788] All rights reserved.

Manuscript submitted January 30, 2006; revised manuscript received March 10, 2006. Available electronically May 4, 2006.

The crystallization of amorphous silicon $(\alpha-\mathrm{Si})$ has been intensively investigated for application in active-matrix flat-panel displays and three-dimensional (3D) electronics. ${ }^{1-10}$ Several techniques have been studied for the fabrication of high-quality polycrystalline silicon (poly-Si) with large crystallite size, low grain boundary defect density, and high carrier velocity. These include excimer laser crystallization (ELC), ${ }^{1-3}$ solid phase crystallization (SPC), ${ }^{4,5}$ and metal induced crystallization (MIC) ${ }^{6,7}$ To obtain large grain polycrystalline silicon with better control over the location of the grain boundaries, metal induced lateral crystallization (MILC) has been widely studied. ${ }^{8-10}$ However, this approach has the disadvantage of introducing metal contamination into the $\alpha-\mathrm{Si}$, with deleterious effects on transistor performance. ${ }^{11}$

Germanium has also been proposed as a seeding agent for the crystallization of $\alpha-\mathrm{Si}^{12,13}$ because of its better compatibility with scaled complementary metal oxide semiconductor (CMOS) technology. The lower melting point of germanium than that of silicon was reported to reduce the incubation time for the crystallization of the underlying amorphous silicon film by the formation of a SiGe layer at the Ge-Si interface. ${ }^{12,13}$ Atomic force microscopy and transmission electron microscopy (TEM) showed increased amorphous silicon crystallization underneath the germanium seed compared with unseeded areas. ${ }^{12,13}$ Improved mobility values were obtained for thin film transistors fabricated in $\alpha$-Si layers crystallized using a germanium seed. ${ }^{12,13}$

In this article we report a new $\alpha$-Si crystallization phenomenon that occurs at the perimeter of a germanium seed during an anneal at $500^{\circ} \mathrm{C}$. This perimeter crystallization phenomenon contrasts with the behavior observed during a $550^{\circ} \mathrm{C}$ anneal where an increased crystallization is seen in all regions beneath the germanium seed, as reported previously. ${ }^{12,13}$ These results suggest the presence of an additional crystallization mechanism in the early stages of the crystallization process; we speculate that this may be due to stress at the perimeter of the germanium seed.

After the growth of $100 \mathrm{~nm}$ thermal oxide at $1100^{\circ} \mathrm{C}$ on (100)-oriented silicon wafers, a $100 \mathrm{~nm}$ undoped LPCVD $\alpha$-Si film was deposited using the pyrolysis of silane at $560^{\circ} \mathrm{C}$. A $300 \mathrm{~nm}$ low pressure chemical vapor deposition (LPCVD) $\mathrm{SiO}_{2}$ layer was then deposited for use as a masking layer, and seeding windows opened using a wet etch. Before germanium deposition, the surface was prepared by a $20 \mathrm{~s}$ dip in a 100:1 HF solution, a water rinse and a spin dry. A $1.6 \mu \mathrm{m}$ polycrystalline germanium layer was then deposited by LPCVD at a temperature of $520^{\circ} \mathrm{C}$ and a pressure of 2 Torr. The wafers were then annealed in argon at $500^{\circ} \mathrm{C}$ for 20,40 and $60 \mathrm{~h}$ or at $550^{\circ} \mathrm{C}$ for $60 \mathrm{~h}$ to laterally crystallize the $\alpha-\mathrm{Si}$.

Samples were prepared for inspection by removing the germa-

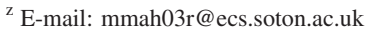

nium layer in an RCA solution and the oxide in HF. The samples were then etched in a $\mathrm{HF}: \mathrm{H}_{2} \mathrm{O}: \mathrm{HNO}_{3}: \mathrm{CH}_{3} \mathrm{COOH}=1: 9: 50: 50$ solution, which selectively etches $\alpha$-Si over poly-Si with a selectivity of over 100:1. Plan-view scanning electron microscopy (SEM) images of these samples were taken. For some samples cross-sectional SEM images were taken after an RCA cleaning immediately followed by an etching for $40 \mathrm{~s}$ in a HF: $\mathrm{HNO}_{3}(1: 400)$ solution to delineate the grain structure in the laterally crystallized $\alpha-\mathrm{Si}$. To analyze further the detailed structure of the laterally crystallized $\alpha-\mathrm{Si}$, samples for plan-view TEM were prepared. A classical preparation method including first a mechanical polishing from the substrate side down to $30 \mu \mathrm{m}$, followed by mechanical fixation of sample pieces in standard $3 \mathrm{~mm}$ ring. The final thinning down to transparency to an electron beam was performed using a Baltec ion milling machine.

Figure 1a shows a top view SEM micrograph of a germaniumseeded sample annealed at $550^{\circ} \mathrm{C}$ for $60 \mathrm{~h}$. The sample was selectively etched to remove any remaining $\alpha-\mathrm{Si}$ and delineate areas of crystallized polysilicon. The $\alpha$-Si beneath the circular germanium seed $(5.62 \mu \mathrm{m}$ diam) has completely crystallized whereas the $\alpha$-Si outside the germanium seed is in the early stages of crystallization. The dark areas indicate where uncrystallized $\alpha-S i$ has been selectively etched and the bright areas indicate where random crystallization has occurred. These results indicate increased crystallization of amorphous silicon beneath the germanium seed compared to the areas without any germanium seed, as has been reported previously. ${ }^{12,13}$

Figure $1 \mathrm{~b}$ shows a top-view SEM of a selectively etched germanium-seeded sample annealed at $500^{\circ} \mathrm{C}$ for $40 \mathrm{~h}$. Dramatically different behavior is observed, with the bright ring around the perimeter of the germanium seed showing laterally crystallized $\alpha$-Si originating from the perimeter of the circular germanium seed. The perimeter crystallization is reasonably uniform, with the crystallization distance varying from a minimum of $220 \mathrm{~nm}$ to a maximum of $510 \mathrm{~nm}$. The average lateral crystallization distance was estimated from measurements on 60 points on six rings and found to have a mean value of $310 \mathrm{~nm}$ and a standard deviation of $\pm 73 \mathrm{~nm}$. The bright specks, both inside and outside the laterally crystallized ring, are due to the random crystallization of individual grains within the amorphous matrix. The density of randomly crystallized grains beneath the germanium seed layer is $(10-15) / \mu \mathrm{m}^{2}$, which is very similar to the density of $(12-16) / \mu \mathrm{m}^{2}$ in regions outside the germanium seed area. These results indicate that there is no evidence of increased grain nucleation beneath the centre of the germanium seed for these anneal conditions, but the lateral crystallization of the $\alpha$-Si occurs at the perimeter of the germanium seed.

Figure 2 shows a cross-sectional SEM of a sample annealed at $500^{\circ} \mathrm{C}$ for $40 \mathrm{~h}$ at a location close to the perimeter of the germanium seed. This sample was not given the selective etch to remove the $\alpha-$ Si layer and hence the uncrystallized $\alpha$-Si can be seen on the left 

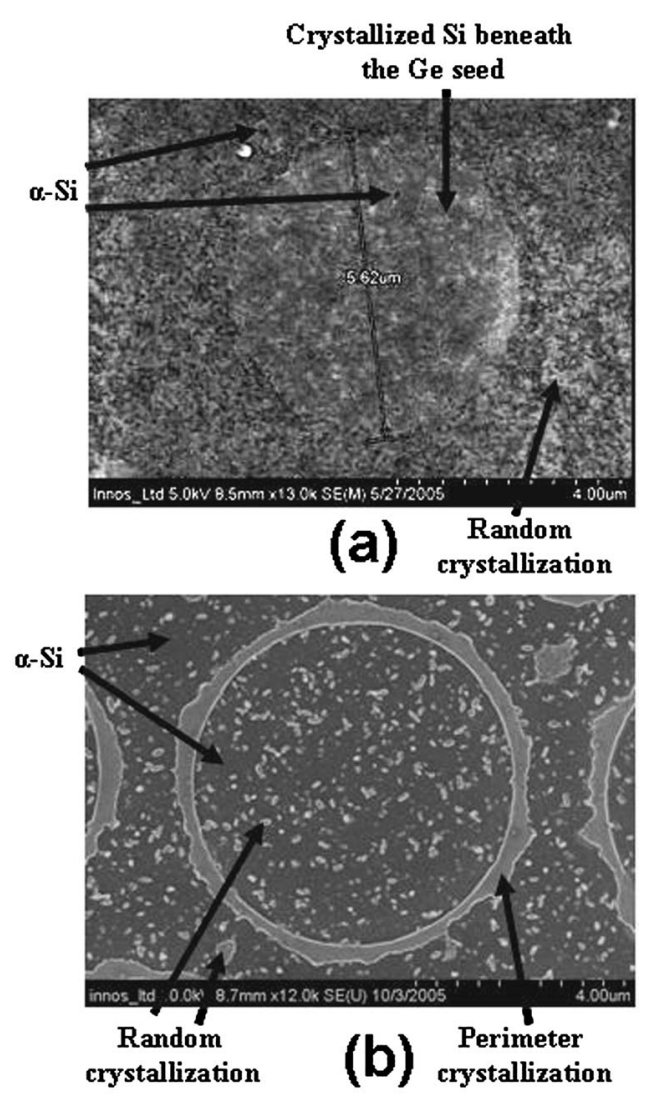

Figure 1. Plan-view SEM micrograph of laterally crystallized amorphous silicon close to the germanium seed layer after an anneal at (a) $550^{\circ} \mathrm{C}$ for 60 and (b) $500^{\circ} \mathrm{C}$ for $40 \mathrm{~h}$. The $100 \mathrm{~nm}$ amorphous silicon layer was undoped and was selectively etched to remove the uncrystallized amorphous silicon.

of the image. The seeded zone, where the germanium seed layer was located can also be seen as a bright contrast on the top right of the image. Lateral crystallization has occurred at the perimeter of the seeded zone, with the lateral grain crystallization occurring both outside and beneath the germanium seed at the perimeter. Within the laterally crystallized region, the crystallization is well advanced at the top surface of the film, but much less complete at the bottom surface, as can be seen from the many isolated islands of $\alpha$-Si at the interface with the bottom $\mathrm{SiO}_{2}$ layer. This behavior was observed on many cross-sectional SEM images and hence is typical of the germanium seeded lateral crystallization process. Away from the germanium seed, on the left of the image, isolated single-crystal grains can be seen, primarily at the bottom $\alpha-\mathrm{Si} / \mathrm{SiO}_{2}$ interface. These

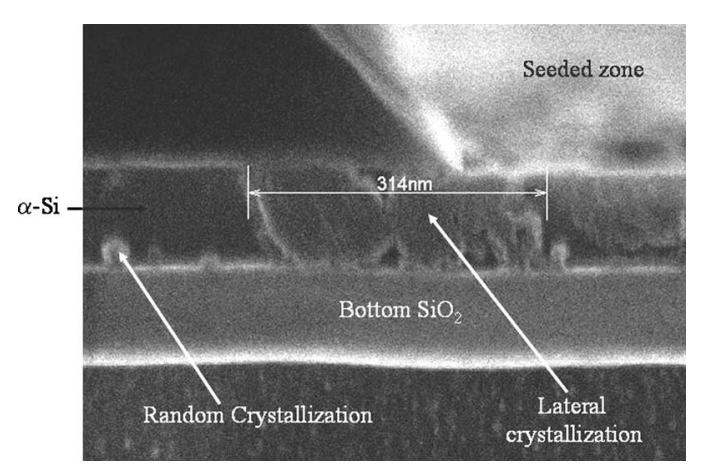

Figure 2. Cross-sectional SEM of a $100 \mathrm{~nm}$ undoped silicon film closed to the seed point annealed at $500^{\circ} \mathrm{C}$ for $40 \mathrm{~h}$.

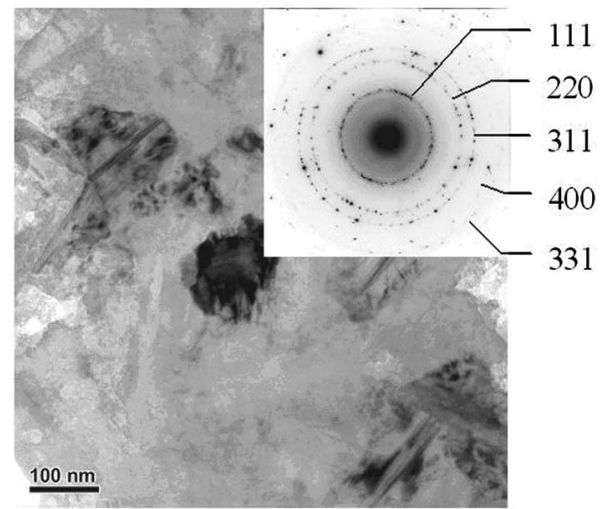

Figure 3. A bright-field plan view TEM image and selected area diffraction patterns of a laterally crystallized region annealed at $500^{\circ} \mathrm{C}$ for $40 \mathrm{~h}$.

grains correspond to the bright specks seen in Fig. 1 outside the laterally crystallized ring and are due to the random nucleation of grains.

To further study the lateral crystallization, Fig. 3 shows a brightfield, plan-view TEM image of a laterally crystallized region adjacent to the germanium seed after the selective etch annealed at $500^{\circ} \mathrm{C}$ for $40 \mathrm{~h}$. The laterally crystallized $\alpha-\mathrm{Si}$ around the perimeter of the germanium seed is made up of polycrystalline silicon with grains of various orientations, as can be seen from the selective area diffraction pattern in Fig. 3 (image insert). The characteristic grain size is close to $100 \mathrm{~nm}$. Numerous structural defects are clearly visible. The diffuse rings in the diffraction pattern are due to the underlying $\mathrm{SiO}_{2}$ which is amorphous.

Figure 4 shows the average and the standard deviation of the lateral crystallization distance as a function of anneal time for samples annealed at $500^{\circ} \mathrm{C}$ for different times. The lateral crystallization distance increases with increasing anneal time and approaches a value of $500 \mathrm{~nm}$ after $60 \mathrm{~h}$ of annealing. For 40 and $60 \mathrm{~h}$ anneals, lateral crystallization is continuous around the perimeter of the germanium seed, whereas for a $20 \mathrm{~h}$ anneal it is discontinuous.

The results in Fig. 1a show increased $\alpha$-Si crystallization beneath the germanium seed after a $60 \mathrm{~h}$ annealing at $550^{\circ} \mathrm{C}$. Similar results were reported by Subramanian et al. ${ }^{12,13}$ and explained by the formation of a $\mathrm{SiGe}$ layer at the $\mathrm{Ge} / \mathrm{Si}$ interface, which reduced the incubation time for the crystallization of the underlying $\alpha-\mathrm{Si}^{14}{ }^{14} \mathrm{It}$ was argued that the grains formed beneath the seeded region and then lateral crystallization occurred when these grains extended into the still amorphous matrix of the unseeded region.

In our work, we observe surprisingly different crystallization behavior after annealing at $500^{\circ} \mathrm{C}$ for $40 \mathrm{~h}$ as shown in Fig. 1b. We find a laterally crystallized ring around the perimeter of the germa-

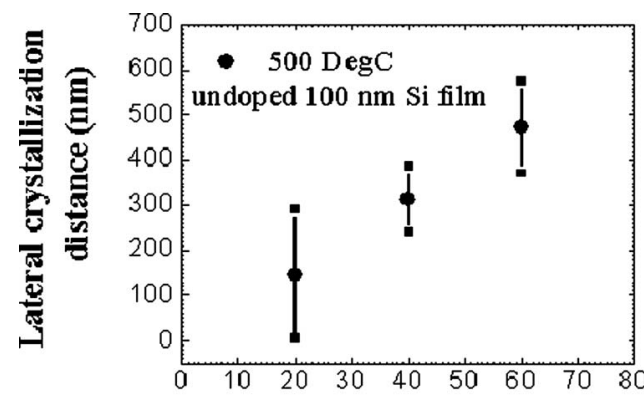

Annealing time(hours)

Figure 4. Lateral crystallization distance, as a function of anneal time for a $100 \mathrm{~nm}$ undoped sample annealed at $500^{\circ} \mathrm{C}$. 
nium seed, with crystallization occurring at all points on the seed perimeter. For these anneal conditions, there is no evidence of increased grain nucleation under the germanium seed, as can be seen from the similar densities of randomly nucleated grains beneath the germanium seed layer $\left(10-15 / \mu \mathrm{m}^{2}\right)$ and in unseeded regions $\left(12-16 / \mu \mathrm{m}^{2}\right)$ in Fig. 1b. Therefore, it can be argued that the origin of the lateral crystallization for these anneal conditions is the perimeter of the germanium seed, which implies a different mechanism than that reported by Subramanian et al. ${ }^{12,13}$ With increasing annealing time at $500^{\circ} \mathrm{C}$ this laterally crystallized region at the perimeter grows laterally until it is retarded by randomly growing crystals in the seeded or unseeded zones as shown in Fig. 4. The grain growth rate has been calculated from the time dependent crystallization around the perimeter of the germanium seed and found to be $\sim 1.4 \mathrm{~A} \%$ min a temperature of $500^{\circ} \mathrm{C}$. This compares with a theoretical growth rate for a completely amorphous silicon film at this temperature of around $0.025 \mathrm{~A}^{\circ} / \mathrm{min}^{15}$ This result implies a significant increase in the grain growth rate around the perimeter of the germanium seed, which supports the argument that this is a different mechanism than reported previously. ${ }^{12,13}$ The location of the $\alpha$-Si crystallization at the perimeter of the germanium seed and the increased grain growth rate might suggest that stress plays a role in inducing lateral crystallization. This might be related to the difference in coefficient of thermal expansion for $\mathrm{Si}$ and $\mathrm{Ge}$ (2.5 compared with $6 \mu \mathrm{m} / \mathrm{m} \mathrm{K}$ ). Tensile stress has also been reported to increase the growth rate of crystallized silicon. ${ }^{16}$ Further experimental work is currently underway to investigate this possibility. This perimeter crystallization phenomenon is advantageous because it gives $\alpha$-Si crystallization in desired locations using a lower thermal budget (typically $40 \mathrm{~h}$ at $500^{\circ} \mathrm{C}$ ) than is obtained when the crystallization occurs beneath the germanium seed (typically $60 \mathrm{~h}$ at $\left.550^{\circ} \mathrm{C}\right)$.

In summary, we have reported a perimeter crystallization phe- nomenon around a germanium seed during a solid phase crystallization anneal at $500^{\circ} \mathrm{C}$. The lateral crystallization is identified to initiate from the perimeter of the germanium seed and is reasonably uniform after annealing at $500^{\circ} \mathrm{C}$ for $40 \mathrm{~h}$, showing lateral crystallization at all points around the perimeter of the seed. With increasing anneal time at $500^{\circ} \mathrm{C}$ the lateral crystallization distance in creases, reaching $500 \mathrm{~nm}$ after $60 \mathrm{~h}$ of annealing. The method is fully CMOS compatible and hence would be attractive for $3 \mathrm{D}$ CMOS integration and large area electronics.

\section{Acknowledgments}

The authors acknowledge the support of the Commonwealth High Commission and European Union (SINANO).

\section{References}

1. T. Sameshima, S. Usui, and M. Sekiya, IEEE Electron Device Lett., 7, 276 (1986). 2. H. J. Kim and J. S. Im, Appl. Phys. Lett., 68, 1513 (1996).

3. R. Ishihara, Y. Hiroshima, D. Abe, B. D. van Dijk, P. C. van der Wilt, S. Higashi, S. Inoue, T. Shimoda, J. W. Metselaar, and C. I. M. Beenakker, IEEE Trans. Electron Devices, 51, 500 (2004).

4. M.-K. Ryu, J.-Y. Kwon, and K.-B. Kim, Mater. Res. Soc. Symp. Proc., 621, Q6.3.1 (2000).

5. T. Noma, T. Yonehara, and H. Kumomi, Appl. Phys. Lett., 59, 653 (1991).

6. C. Hayzelden and J. L. Batstone, J. Appl. Phys., 73, 8279 (1993).

7. Z. Jin, G. A. Bhat, M. Yeung, H. S. Kwok, and M. Wong, J. Appl. Phys., 84, 194 (1998).

8. S.-W. Lee and S.-K. Joo, IEEE Electron Device Lett., 17, 160 (1996).

9. A. R. Joshi and K. C. Sarasawat, J. Electrochem. Soc., 150, G443 (2003)

10. Z. Meng, M. Wang, and M. Wong, IEEE Trans. Electron Devices, 47, 404 (2000).

11. S. Naito and T. Nakashizu, in Defect Engineering in Semiconductor Growth, Processing, and Device Technology, p. 641, MRS, Warrendale, PA (1992).

12. V. Subramanian and K. C. Saraswat, IEEE Trans. Electron Devices, 45, 1934 (1998).

13. V. Subramanian, M. Toita, N. R. Ibrahim, S. J. Souri, and K. C. Saraswat, IEEE Electron Device Lett., 20, 341 (1999).

14. J. H. Kim, J. Y. Lee, H. S. Kim, Y.-H. Song, and K.-S. Nam, IEEE Electron Device Lett., 17, 205 (1996).

15. N. Yamauchi and R. Reif, J. Appl. Phys., 75, 3235 (1994).

16. C.-Y. Hou and Y. S. Wu, Jpn. J. Appl. Phys., Part 1, 44, 7327 (2005). 